\title{
Penalizing Source Uncertainty in Wholesale Electricity Markets
}

\author{
D. Georgiev and E. Janeček
}

\begin{abstract}
Many renewable energy sources are intermittent. The DC optimal power flow method used by wholesale markets for economic dispatch and energy pricing provides no way of evaluating the worth of such sources. We propose that the bids of the intermittent sources include uncertainty levels as well as energy prices, and develop, for radial distribution networks, a new probabilistic version of the DC OPF problem that bears the same structure but penalizes intermittency. The new problem is solved for several instances of the IEEE 32 node radial network. It is shown that the probabilistic approach correctly captures many economic and operational effects of source uncertainty. While fixed energy sources have a greater value at distant locations, intermittent energy sources are better near fixed energy sources that can compensate their power fluctuations.
\end{abstract}

\section{INTRODUCTION}

$\mathbf{T}$ HE global effort to curb carbon emissions has led goverments to propose greater renewable energy utilization targets [1]. Greater integration of renewable energy sources (RESs) into wholesale electricity markets (such as the Czech OTE) is a key to meeting these targets.

It is known that many RESs are intermittent [2], [3], which means that, in the day ahead market and the real time market, their bids can only be given probabilistically. Majority of wholesale markets use the DC optimal power flow (OPF) problem to allocate the energy sources [4], i.e., for economic dispatch (ED), and to calculate the market clearing energy prices, called the least marginal prices (LMPs). The DC OPF problem, however, provides no way of dealing with probabilistic energy bids. Studies have only considered load uncertainty for price sensitivity reduction (see [5]) and compensation/penalties for normalization of real-time deviations and robust energy trading motivation (see [6]). As a result, intermittent energy sources (IESs) are not allowed to bid in the day ahead market and are required to abide by special rules in the real time market [2]. The real time market rules suggest an effort to force IESs to behave consistently with the DC OPF problem formulation. Herein, we take an alternate approach and develop a probabilistic DC OPF (PDC OPF) problem that penalizes intermittency in a fair way according to its level and location.

The underlying idea is for intermittent energy sources to bid uncertainty coefficients, which link their power variances to their power means, together with the price bids to the ISO,

This work was supported in part by the grants WAMS-MPO FR-TT1/462, TA ČR TA01020865, and CAK-MŠMT 1M0567.

D. Georgiev and E. Janeček are with the Department of Cybernetics in the College of Applied Sciences, University of West Bohemia, Pilsen, 30000, Czech Republic. janecek@kky.zcu.cz, georgiev@kky.zcu.cz which then uses these bids in the PDC OPF problem derived herein.

It is our belief that this approach will lead to a more efficient/robust ED and LMPs that are more reflective of the actual energy delivery cost. Both consequences should enable ISOs to better integrate IESs into their day ahead and real time markets even at greater penetration levels. The following helpful effects were observed in a study of the radial IEEE 32 node distribution network.

1. Source uncertainty increases the LMPs reflecting the true cost of energy delivery.

2. Source uncertainty increases overall power generation leading to a more robust dispatch and a lesser need for regulation reserves.

3. The resulting ED naturally balances dispatch from local IESs with non-local fixed energy sources, which means that IESs are only used when they're economically favorable.

4. IESs closer to fixed energy sources are more utilized creating a stabilizing market force that motivates a more robust distributed energy source topology.

The remainder of the paper is organized as follows: The network model with the necessary assumptions is presented in Section II. The key new component of the PDC OPF, uncertainty losses, are derived in Section III. Uncertainty losses are integrated into the power flow equations to formulate the PDC OPF problem in Section IV. The method is tested on the radial IEEE 32 node network in Section V. The paper concludes with Section VI.

\section{Network MOdel}

The following notation is used throughout. Capital letters are used to denote random variables and matrices. The letter $i$ is reserved for the imaginary unit. Any vector $\left(x_{1}, \ldots, x_{n}\right)$ can be simply written as $\left(x_{k}\right)_{k \in\{1, \ldots, n\}}$ or as $x$. For random variables $X$, the expectation value is written as $\langle X\rangle$ and the variance is written as $\langle\langle X\rangle\rangle$.

Distribution networks often have a highly radial structure. For simplicity we assume that the network is purely radial. Future work will consider non-radial structures. A radial network topology is equivalent to a directed tree graph. The root node corresponds to the power source and the other nodes correspond to the customers. The branches represent the power lines and are directed downstream towards the power source. The following is a formal description.

Consider a directed graph where the set of nodes is given by the finite set $\mathcal{N}=0,1, \ldots, N$ and the set of branches is defined 
by the set $\mathcal{B} \subset \mathcal{N} \times \mathcal{N}$, where each $(k, \ell) \in \mathcal{B}$ is directed from node $k$ to node $\ell$. For each node $k \in \mathcal{N}$, define the set of upstream nodes by the set $\mathcal{U}_{k}=\{j \in \mathcal{N} \mid \exists(j, k) \in \mathcal{B}\}$. Any node in the graph that has an empty set of upstream nodes is called a leaf node. If there exists a path from node $k$ to node $\ell$, than $k$ is said to be upstream of $\ell$ (denoted by $k>\ell$ ) and $\ell$ is said to be downstream of $k$ (denoted by $\ell<k$ ).

The network is considered at a specific instant in time where the graph nodes and branches are associated with the following network variables. For each node $k \in \mathcal{N}$, denote the loop current by $J(k)$, the load by $p_{L}(k)$, and the generated power by $P_{G}(k)$. For the branch $b=(k, \ell) \in \mathcal{B}$, denote the impedance by $z(k)=r(k)+i x(k)$, the current by $I(k)$, the power losses by $S(k)$, and the power transmitted to the branch from node $k$ by $F(k)$.

The following is the list of assumptions used in our work:

A1. the network is radial with node 0 at the root, i.e., the set $\mathcal{B}$ is such that there exists a single path (defined as a directed connection of edges) leading to node 0 from every other node,

A2. the network topology and branch impedances are fixed and known, the network is balanced and operating under normal conditions with no failures and filled power demands,

A3. the voltage at node 0 has a fixed magnitude $v_{0}$ and a fixed phase,

A4. the loads $p_{L}{ }^{1}$ are known,

A5. if power source $k$ supplies a mean power of $\left\langle P_{G}(k)\right\rangle$ units, then the variance of the supplied power is $v_{k}\left(\left\langle P_{G}(k)\right\rangle\right)$,

A6. the loop currents $J$ are mutually independent complex random variables ${ }^{2}$,

A7. the voltage drop is negligible, i.e., for all $k \in \mathcal{N}$, the magnitude of the node voltage is $v_{0}$,

A8. the probability of a large deviation of any nodal voltage phase angle is small, i.e., for all nodes $k \in \mathcal{N}$, if the voltage phase is $\Theta(k)$, then the probability of $\left\{\left|\Theta_{k}-\left\langle\Theta_{k}\right\rangle\right|<\epsilon\right\}$ is approximately one.

Distribution networks often have a highly radial structure. For simplicity we assume the structure is purely radial (Assumption 1). By Assumption 2, we can treat the network as a single phase system, where the nodes correspond to the buses and branches correspond to the lines, transformers, reactors, etc [4]. Assumption 3 is standard in the DC OPF framework, it simply sets the location of the base power source that ensures all the power demands are met regardless of the distributed generator capacities [4]. Assumption 7 is also standard in the DC OPF framework. Assumption 8 is justifiable in the DC OPF framework which assumes small phase angle differences between node voltages and a fixed voltage at node 0 .

\footnotetext{
${ }^{1}$ Computations of LMP prices and power allocation typically occur every hour. The load serving entities state exactly their load requirements for that hour.

${ }^{2}$ Weather effects may correlate generation. The treatment herein neither rewards nor penalizes the sources for such chance events.
}

\section{UNCERTAINTY LOSSES}

For any branch $k \in \mathcal{N}$, the expected value of the branch loss is a sum of deterministic and uncertain components, i.e.,

$$
\langle S(k)\rangle:=z(k)\left\langle I(k) I^{*}(k)\right\rangle=s_{D}(k)+s_{U}(k) .
$$

Supplemental methods that estimate the deterministic loss component for the lossless DC OPF problem exist [7]. Hence, the novelty of this work is the consideration of the uncertainty losses $s_{U}$. In this section, we show the deterministic component $s_{D}$ is a function of the expected power flow across the branch and the uncertainty component $s_{U}$ is a function of the upstream generated power variances. In the next section, we use $s_{U}$ to formulate the PDC OPF problem.

Consider the expectation

$$
\left\langle I(k) I^{*}(k)\right\rangle=\left\langle|I(k)|^{2}\right\rangle=\langle|I(k)|\rangle^{2}+\langle\langle|I(k)|\rangle\rangle .
$$

By Assumption 7, $|I(k)|=|F(k)| / v_{0}(F(k)$ is the power transmitted from node $k$ ). By Assumption 8, the deviations of the angle of $I(k)$ are small, which implies that $\langle\langle|I(k)|\rangle\rangle=$ $\langle\langle I(k)\rangle\rangle$. Lastly, by Assumption 6, $\langle\langle I(k)\rangle\rangle=\sum_{\ell \geq k}\langle\langle J(\ell)\rangle\rangle$. Hence

$$
\left\langle I(k) I^{*}(k)\right\rangle=\left\langle|F(k)| / v_{0}\right\rangle^{2}+\sum_{\ell \geq k}\langle\langle J(\ell)\rangle\rangle .
$$

Deviations of the angle of $J(\ell)$ are small, since, by Kirchoff's current law, it is linearly dependent on branch currents whose angle deviations are small by assumption. Therefore, its variance is also equal to the variance of its norm. The norm of $J(k)$ is related to the generated power at node $k$ by the equation $|J(k)|=\left|P_{G}(k)\right| / v_{0}$. Together, these fact combine to produce the final expression for the deterministic and uncertainty losses.

$$
\begin{aligned}
s(k) & =\frac{z(k)}{v_{0}^{2}}\left(\langle|F(k)|\rangle^{2}+\sum_{\ell \geq k}\left\langle\left\langle\left|P_{G}(\ell)\right|\right\rangle\right\rangle\right) \\
& =\frac{z(k)}{v_{0}^{2}}\left(\langle|F(k)|\rangle^{2}+\sum_{\ell \geq k}\left\langle\left\langle P_{G}(k)\right\rangle\right\rangle\right),
\end{aligned}
$$

where the last equation follows since the angle deviations of $P_{G}(k)-p_{L}(k)$ can also be shown to be small.

It is common practice in economic dispatch and market clearing problems to work with dimensionless variables [4]. The power, impedance, and voltage scaling factors are related by $p_{0}=v_{0}^{2} / z_{0}^{2}$. Scaling the loss equation by $p_{0}$ and utilizing the definition of the variance function $v_{k}$ yields

$$
s^{p u}(k)=z^{p u}(k)\left(\left\langle\left|F^{p u}(k)\right|\right\rangle^{2}+\sum_{\ell \geq k} v\left(\left\langle P_{G}^{p u}(k)\right\rangle\right)\right) .
$$

\section{PROBABILISTIC DC OPF}

In this section we assume that all variables and parameters are given in the pu scale (eliminating the need for the pu superscript). To further simplify the exposition, the expected values of all random variables are denoted by the same letters in lower case. 
The assumptions of the DC OPF formulation comprise Assumptions 2, 3, and 7, as well as small phase differences in node voltage angles, null resistances for all branches, and null reactive power demand and generation [4]. Such networks are lossless. So, to more accurately model the true energy delivery costs, branch losses are added to the idealized power flow equations to yield the standard problem [8] (simplified for radial networks).

\section{Problem (Standard DC OPF).}

$$
\underset{F, P_{G}}{\operatorname{minimize}} \sum_{k \in \mathcal{N}} a(k) P_{G}(k)+b(k)\left(P_{G}(k)^{2}\right),
$$

subject to,

$$
\begin{aligned}
& p_{L}(k)=P_{G}(k)-F(k)+\sum_{\ell \in \mathcal{U}_{k}} F(\ell)-S(\ell): \Lambda_{\text {cost }}(k), \\
& |F(k)| \leq f_{\max }(k): \Lambda_{\text {cong }}(k), \\
& p_{\min }(k) \leq P_{G}(k) \leq p_{\max }(k), \quad F(0)=0,
\end{aligned}
$$

where $k \in \mathcal{N}$ and $\Lambda_{\text {cost }}$ and $\Lambda_{\text {cong }}$ represent the corresponding Lagrange multipliers that sum to give the LMPs.

Under our formulation, all variables above in capitol letters are random. If we take their expected values, denoted by the same letters in lower case, the problem is essentially unchanged except for the quadratic terms in the cost function and the loss expressions. Substituting the results from the previous section, with all generated and load reactive powers set to zero, yields the following new problem (written for radial networks only).

\section{Problem (Probabilistic DC OPF).}

$$
\underset{f, p_{G}}{\operatorname{minimize}} \sum_{k \in \mathcal{N}} a(k) p_{G}(k)+b(k)\left(p_{G}(k)^{2}+v_{k}\left(p_{G}(k)\right)\right),
$$

subject to,

$$
\begin{aligned}
& p_{L}(k)=p_{G}(k)-f(k) \\
& +\sum_{\ell \in \mathcal{U}_{k}} f(\ell)-r(\ell)\left(f(\ell)^{2}+h(\ell)\right): \lambda_{\text {cost }}(k) \\
& |f(k)| \leq f_{\max }(k): \lambda_{\text {cong }}(k), \\
& p_{\min }(k) \leq p_{G}(k) \leq p_{\max }(k), \\
& h(k)=\sum_{\ell \in \mathcal{U}_{k}} h(\ell)+v_{\ell}\left(p_{G}(\ell)\right), \\
& v_{0}\left(p_{g}(0)\right)=h(1), \quad f(0)=0,
\end{aligned}
$$

where $k \in \mathcal{N}$, and $\lambda_{\text {cost }}$ and $\lambda_{\text {cong }}$ are the corresponding Lagrange multipliers so that $L M P(k)=\lambda_{\text {cost }}(k)+\lambda_{\text {cong }}(k)$.

Note that this problem is not convex due to the quadratic loss terms in the power flow equality constraints. When considering deterministic losses, the common practice is to achieve convexity through linearization. We proceed similarly by approximating the deterministic and uncertain losses by

$$
s_{D}(k)=r(k) w(k)|f(k)|, \quad s_{U}(k)=r(k) \sigma(k) \sum_{\ell \geq k} p_{G}(\ell) .
$$

Other approximations of deterministic losses may be used [7]. Implementing these expressions in the PDC OPF problem yields a convex optimization problem with the same complexity as the standard DC OPF problem.

\section{COMPARISON STUDY}

Effects of uncertainty losses on LMPs and ED were studied for a $12.66 \mathrm{kV}$ IEEE 32 node bus network that was first published in [9]. An equally uncertain power source was placed at each node. Active loads published in [9] were also placed at all the nodes, while the reactive power demands were ignored (as is done in the DC formalism).

The study focused on the effects of branch losses and not on congestion effects by removing branch capacity and power generation constraints, i.e., for all $k \in \mathcal{N}, f_{\max }(k)=\infty$ and $p_{\max }(k)=\infty$. Two variations of the convex PDC OPF problem were studied for various levels of uncertainty. In the first variation, deterministic losses were ignored. For $k \in \mathcal{N}$, LMPs and the ED were calculated for the MW values of $\sigma(k) \in\{0.0,0.06,0.12,0.18,0.24\}^{3}$. In the second variation, deterministic losses were included. LMPs and the ED were calculated for the same values of $\sigma$ and, for all $k \in \mathcal{N}$, $w(k)=.12 \mathrm{MW}$. In all calculations, the source energy cost bids $a(k)=1 \$ / M W$ and $b(k)=.1 \$ / M W^{2}$.

The results from the first and second variations of the DC OPF problem are shown in the first and second column, respectively, in Figure 1. Several interesting effects (see Figure 1) were produced by the uncertainty losses with or without the deterministic power losses.

1. Source uncertainty increases the LMPs at all nodes. Uncertainty corresponds to greater losses, which increases the cost of energy.

2. Source uncertainty increases the overall power generated. Additional power is required to compensate the greater losses and meet the demand.

3. The fixed power source supplies a greater fraction of the power as uncertainty increases. Energy from IESs is discounted by their uncertainty and hence traditional fixed sources are preferred.

4. IESs closer to the fixed source are preferred. Intermittency of power sources must be compensated. The farther away the intermittent source is from the fixed source, the more expensive it is to compensate.

Comparing the EDs of the two problem variations revealed how the deterministic and uncertainty losses complement each other. In the absence of the $s_{D}$ term, the fixed power source can deliver energy to any node at the same cost but it must pay a greater cost to compensate intermittency at nodes farther away on the network. As a result, the fixed source slowly replaces the distant IESs as uncertainty increases. This no longer holds in the presence of the $s_{D}$ term; distant IESs continue to be utilized even at greater levels of uncertainty. Based on these observations we conclude the following about the tradeoffs between these two types of branch losses. Deterministic losses promote greater source distribution to decrease the source-toload distance. Uncertainty losses promote locational coupling

${ }^{3}$ The dimensionless values and the MW values of $\sigma(k)$ are related by $\sigma(k)=\sigma^{p u}(k) p_{0}$. These values were chosen by taking percentages of the mean power demand. 


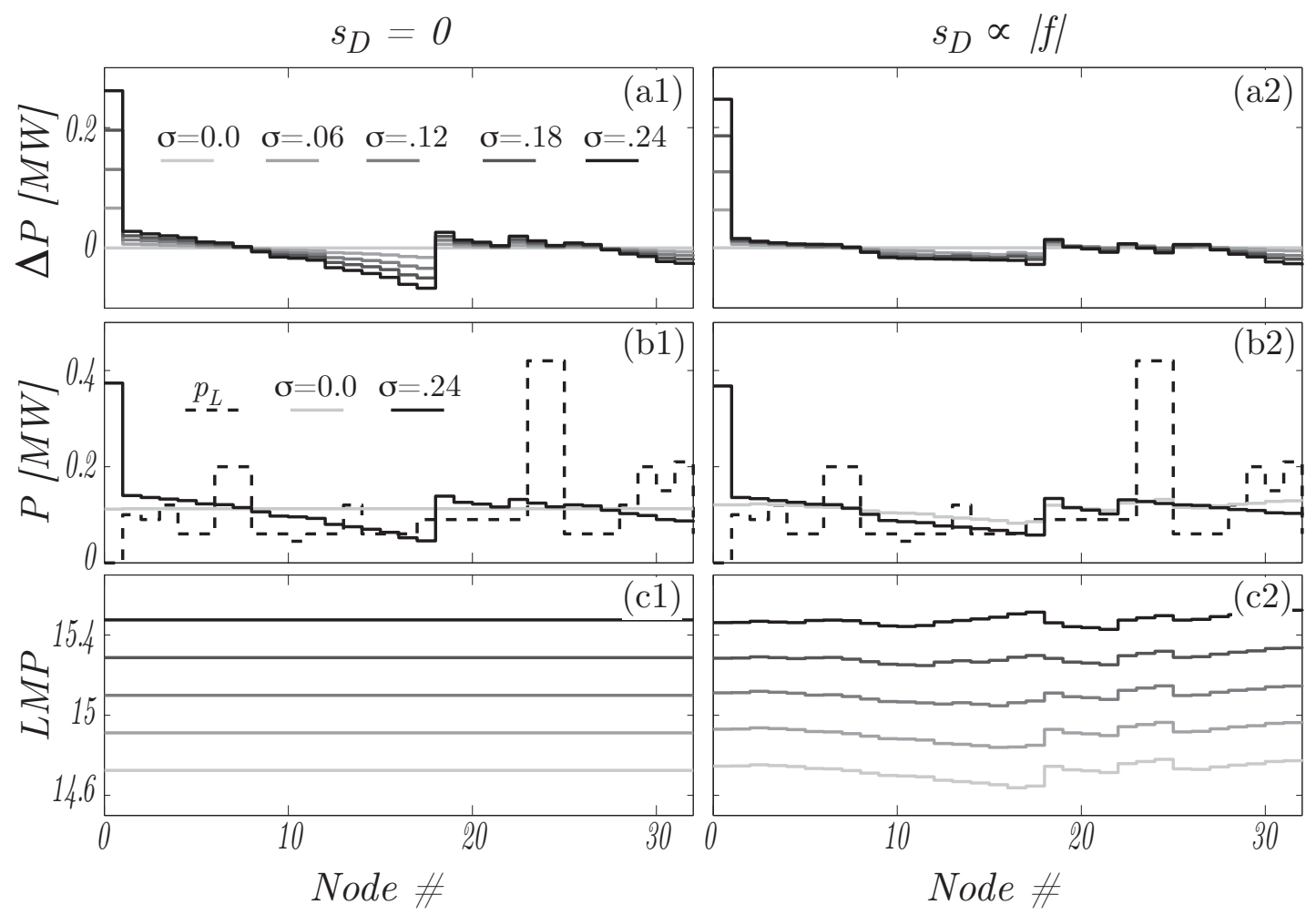

Fig. 1: Economic dispatch and LMP pricing for the IEEE 32 node distribution network illustrating the effect of variance losses. The left column plots include only variance losses while the right column plots includes variance losses and the deterministic losses described in this section. Plots in row (a) illustrate the changes in economic dispatch as uncertainty increases from $\sigma=0.0$ (lightest shade of grey) to $\sigma=0.24 \mathrm{MW}$ (darkest shade of grey). Plots in row (b) illustrate the EDs at the extremal uncertainty levels (light grey for $\sigma=0.0$ and dark grey for $\sigma=0.24$ ) and how they compare to the node loads (dashed). Plots in row (c) illustrate the least marginal prices. The prices reflect only the energy delivery cost as the branch and generator capacity are assumed to be unlimited.

between fixed and intermittent sources to decrease compensation costs by decreasing the source-to-compensator distance.

\section{CONCLUSION}

In this paper we proposed that IESs bid uncertainty coefficients, which link their power variances to their power means, together with their price bids. We then derived a probabilistic DC OPF problem ISOs can use to compute an ED that is more efficient/robust and LMPs that are more reflective of the actual energy delivery cost. In the PDC OPF problem, the powers are treated as random variables and all the constraints in the traditional DC OPF problem are transformed into constraints on expected values. The basic structure of the problem remains the same with new terms present in the flow equations and cost function. It is observed that the PDC OPF naturally balances deterministic losses and uncertainty losses to 1) penalize higher uncertainty, 2) penalize distance to compensating energy sources, and 3) reward close proximity to loads. We believe the PDC OPF problem can yield greater RES penetration by presenting a method for evaluating the worth of their energy (thereby allowing greater participation in the day ahead and real time markets), and by promoting cooperation amongst intermittent and fixed energy sources (thereby motivating more robust network generation topologies).

\section{REFERENCES}

[1] Q. Zhou and J. Bialek, "Generation curtailment to manage voltage constraints in distribution networks," IET Transmission and Distribution, vol. 1, 2007.

[2] A. Botterud, J. Wang, C. Moteiro, and V. Miranda, "Wind power forecasting and electricity market operations," in Proc. of the IAEE Int. Conf., 2009.

[3] J. Zhou, "20\% wind generation and the energy markets: A model and simulation of the effect of wind on the optimal energy portfolio," Master's thesis, Princeton University, Dep. of Operations Research and Financial Engineering, 2010.

[4] J. Sun and L. Tesfatsion, "DC optimal power flow formulation and solution using QuadProgJ," Iowa State University, Tech. Rep., 2010.

[5] R. Bo and F. Li, "Probabilistic LMP forecasting considering load uncertainty," IEEE Trans. on Power Systems, vol. 24, 2009.

[6] P. Attaviriyanupap and A. Yokoyama, "Price volatility and service interruption risk-hedging by transmission contract," IEEE Trans. on Power Systems, vol. 21, 2006.

[7] E. Litvinov, T. Zheng, G. Rosenwald, and P. Shamsollahi, "Marginal loss modeling in LMP calculation," IEEE Trans. on Power Systems, vol. 19, no. 2,2004

[8] T. Alvey, D. Goodwin, X. Ma, D. Streiffert, and D. Sun, "A securityconstrained bid-clearing system for the New Zealand wholesale electricity market," IEEE Trans. on Power Delivery, vol. 13, 1998.

[9] M. E. Baran and F. F. Wu, "Network reconfiguration in distribution systems for loss reduction and load balancing," IEEE Transactions on Power Delivery, vol. 4, 1989. 